\title{
Extract of high hydrostatic pressure-treated danshen (Salvia miltiorrhiza) ameliorates atherosclerosis via autophagy induction
}

\author{
Minjeong $\mathrm{Ko}^{1}$, Goo Taeg Oh${ }^{2}$, Jiyong Park ${ }^{3}$ \& Ho Jeong Kwon ${ }^{1, *}$ \\ ${ }^{1}$ Chemical Genomics Global Research Lab, Department of Biotechnology, College of Life Science and Biotechnology, Yonsei University, \\ Seoul 03722, ${ }^{2}$ Department of Life Sciences, Ewha Womans University, Seoul 03762, ${ }^{3}$ Department of Biotechnology, College of Life Science \\ and Biotechnology, Yonsei University, Seoul 03722, Korea
}

\begin{abstract}
Danshen (Salvia miltiorrhiza) is a traditional medicinal plant widely used in Asian countries for its pharmacological activities (e.g., amelioration of cardiovascular diseases). In this study, we investigated the anti-atherosclerotic activity of raw danshen root extract prepared using high hydrostatic pressure (HHP) at $550 \mathrm{MPa}$ for $5 \mathrm{~min}$ and hot water extraction. This method was useful for elimination of bacteria from cultured danshen plants and for better extraction yield of active principles. The HHPtreated danshen extract (HDE) inhibited proliferation of human umbilical vein endothelial cells (HUVECs) and induced autophagy that was assessed by LC3 conversion and p62 degradation. HDE suppressed foam cell formation in oxLDL-induced RAW264.7 macrophages; lysosomal activity simultaneously increased, measured by acridine orange staining. HDE also reduced atherosclerotic plaque development in vivo in apolipoprotein E knock-out $\left(\mathrm{ApoE}^{-/}\right)$mice fed a high cholesterol diet. Taken together, these results indicated that HDE exhibited anti-atherosclerotic activity via autophagy induction. [BMB Reports 2020; 53(12): 652-657]
\end{abstract}

\section{INTRODUCTION}

Cardiovascular disease, including atherosclerosis, is the number one cause of death worldwide (1). Atherosclerosis is a chronic inflammatory disease in which blood vessels are gradually blocked due to accumulation of cholesterol and plaque formation inside the blood vessel wall. When vascular endothelial cells (ECs) are damaged by stimuli at the beginning of atherosclerosis,

*Corresponding author. Tel: +82-2-2123-5883; Fax: +82-2-362-7265; E-mail: kwonhj@yonsei.ac.kr

https://doi.org/10.5483/BMBRep.2020.53.12.184

Received 1 September 2020, Revised 23 September 2020, Accepted 11 November 2020

Keywords: Atherosclerosis, Autophagy, Cryptotanshinone, High hydrostatic pressure, Salvia miltiorrhiza monocytes attach to blood vessel walls, enter under the ECs, then differentiate into macrophages (2). Macrophages become "foam cells" that absorb oxidized low-density lipoprotein (LDL) and secrete inflammatory cytokines to further increase the inflammatory response. Previous reports suggest that regulation of foam cell formation induced by oxLDL will contribute to prevent atherosclerosis $(3,4)$.

Using a non-thermal high hydrostatic pressure (HHP) method, HHP technology is widely used as a processing technology for food materials $(5,6)$. In addition, ultrahigh pressure treatment is useful for making high quality functional food materials and increasing the extraction efficiency of internal active principles (7-10).

Danshen (Salvia miltiorrhiza) is a popular traditional Chinese medicinal plant that promotes various biological effects (11, 12). The plant includes compounds such as diterpene-containing tanshinone I, IIA, and IIB, cryptotanshinone, and phenolic compounds (e.g., danshensu, protocatechuic aldehyde, and salvianolic acid B) (13). It has been prescribed for vascular diseases (e.g., coronary heart disease, angina pectoris, myocardial infarction, stroke, and blood circulation) in China and other countries in Asia. However, effects of danshen extract on suppression of foam cell formation and alleviation of atherosclerotic plaque growth have not been examined.

Autophagy is a mechanism used to decompose and recycle unnecessary organelles in a cell. It is involved in maintenance of cell homeostasis (14). Dysfunctional proteins can not be degraded when autophagy does not occur normally, leading to diseases such as cancer and degenerative and metabolic diseases (15). Autophagy also plays an important role in regulation of atherosclerosis (16) and malfunctions in macrophage autophagy have been implicated in formation of atherosclerotic plaques (17).

In this study, we found that HHP-treated danshen extract (HDE) has an anti-atherosclerotic effect by reducing foam cell formation through autophagy activation in RAW264.7 cells in vitro and in $A p o E^{-1-}$ mice in vivo.

ISSN: 1976-670X (electronic edition)

Copyright (c) 2020 by the The Korean Society for Biochemistry and Molecular Biology

(c) This is an open-access article distributed under the terms of the Creative Commons Attribution Non-Commercial License (http://creativecommons.org/licenses/by-nc/4.0) which permits unrestricted non-commercial use, distribution, and reproduction in any medium, provided the original work is properly cited. 


\section{RESULTS}

\section{Effects of HHP treatment on the population of microorganisms and extraction of active principles from raw danshen}

Populations of aerobic bacteria, yeasts, and molds were measured to investigate the effects of HHP treatment (550 MPa for 5 min) on microorganisms in raw danshen (Fig. 1B, C). The numbers of total aerobes in raw danshen (control) were approximately $119 \times 10^{3}$ (colony forming units) CFU/g. Pressurization inactivated it to nearly $1.14 \times 10^{3} \mathrm{CFU} / \mathrm{g}$. Likewise, initial yeast and mold counts for control danshen were about $510 \times$ $10^{3} \mathrm{CFU} / \mathrm{g}$, then decreased to nearly $0.18 \times 10^{3} \mathrm{CFU} / \mathrm{g}$ after HHP treatment. These results indicated that HHP treatment effectively eliminated microorganisms on raw danshen in a short time, without color and flavor changes.

HHP treatment destroys cell wall structures and enhances solvent permeability of the cells $(10,18)$. Therefore, HHP treatment of a raw danshen plant facilitates extraction of active principles via increased transfer of intracellular materials to the solvent. Raw danshen and HHP-pretreated danshen were extracted with the conventional boiling water extraction method (Fig. 1A). The average extraction yield of HHP-treated danshen $(0.97 \pm 0.17)$ was about 1.3 times greater than that of raw danshen $(0.72 \pm 0.12)$ (Fig. 1D). This result indicated that HHP treatment effectively increased extraction yield of active principles from raw danshen.

\section{HHP-treated danshen extract induced autophagy flux of human umbilical vein endothelial cells}

To assess the potential of HDE for treatment of cardiovascular disease, we first examined effects of HDE in ECs. The effect of HDE on human umbilical vein endothelial cells (HUVECs) proliferation was investigated using MTT assay. HDE treatment at $0-100 \mu \mathrm{g} / \mathrm{ml}$ suppressed cell proliferation in a dose-dependent manner (Fig. 2A). The $\mathrm{IC}_{50}$ value of $\mathrm{HDE}$ was $34.6 \mu \mathrm{g} / \mathrm{ml}$ and

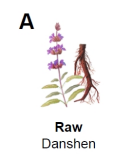

B

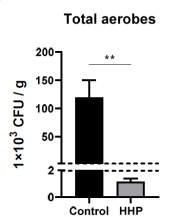

C

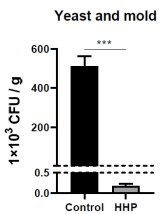

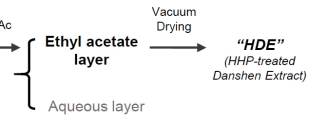

D

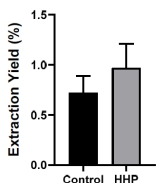

Fig. 1. Effects of HHP treatment on danshen root. (A) Overview of extraction procedures. ( $\mathrm{B}, \mathrm{C})$ Effects of HHP treatment on danshen on the populations of total aerobic bacteria, yeast and mold. (D) Extraction yield increased by about $30 \%$ after HHP treatment. Results are presented as mean \pm standard error of the mean (SEM) values. Statistical significance was assessed using Student's t-tests. $* * * \mathrm{P}<$ $0.001 ; * * P<0.01$ no cytotoxicity was observed.

To investigate the effect of HDE on autophagy flux, the levels of LC3B conversion and p62 expression in HUVECs were assessed over time (Fig. 2B). Treatment of HUVECs with HDE resulted in LC3B-I to LC3B-II conversion and p62 degradation after 24-48 h. This result suggested that HDE induced autophagy that contributed to suppression of HUVECs proliferation. Chloroquine is a lysosome activity inhibitor widely used to suppress autophagy flux. HDE treatment reduced p62 levels, indicating that autophagy was activated. However, the increased p62 levels during chloroquine treatment indicated that autophagy flux inhibition occurred (Fig. 2C). We also found that chloroquine increased upregulation of LC3B-II by HDE. To further confirm the HDE-induced autophagy process, autophagic flux was monitored using a tandem-labeled GFP-mRFP-LC3 system. GFP is sensitive to an acidic $\mathrm{pH}$ level; when it is fused with lysosomes, green fluorescence is quenched and red fluorescence is remained (19). Thus, in this assay, autophagosomes were labeled

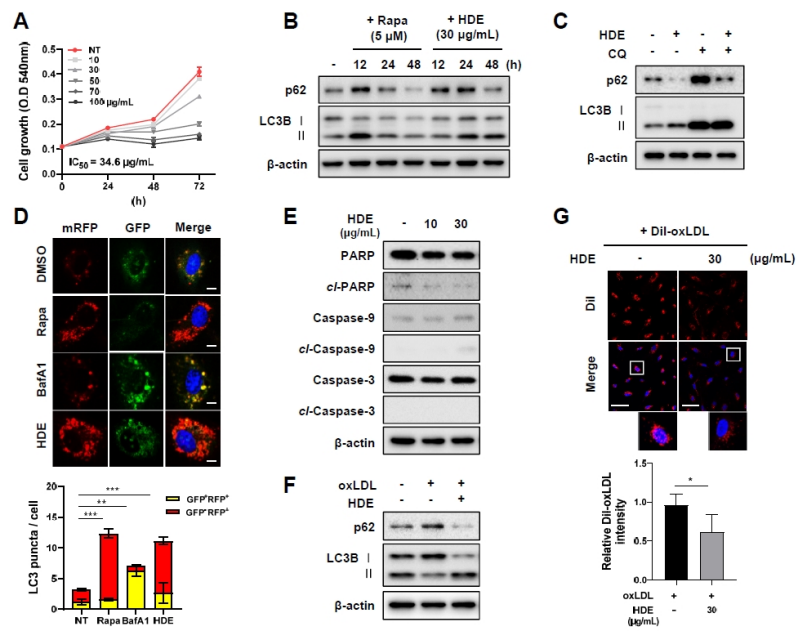

Fig. 2. HDE inhibited cell proliferation and induced autophagy in HUVECs. (A) MTT assay was performed to analyze the effect of HDE on cell proliferation. HDE decreased cell proliferation in HUVECs in a concentration-dependent manner $(0-100 \mu \mathrm{g} / \mu \mathrm{l})$ for $72 \mathrm{~h}$. (B) Autophagy was monitored on western blots of protein lysates of HUVECs following $30 \mu \mathrm{g} / \mathrm{ml} \mathrm{HDE}$ treatments at 12, 24, and $48 \mathrm{~h}$. (C) Cells were pretreated with chloroquine $(25 \mu \mathrm{M})$ (or were not pretreated) for $1 \mathrm{~h}$, before incubation in the presence or absence of $30 \mu \mathrm{g} / \mathrm{ml}$ HDE for 48 h. (D) HUVECs were transiently transfected with GFPmRFP-LC3 and treated with rapamycin $(10 \mu \mathrm{M})$ for $12 \mathrm{~h}$, BafA1 $(10 \mathrm{nM})$ for $3 \mathrm{~h}$, or HDE $(30 \mu \mathrm{g} / \mathrm{ml})$ for $48 \mathrm{~h}$, and then examined for changes in green and red fluorescence using a confocal microscope (Scale bar: $10 \mu \mathrm{m})$. The numbers of red puncta $\left(\mathrm{GFP}^{-} \mathrm{RFP}^{+}\right.$) versus yellow puncta $\left(\mathrm{GFP}^{+} \mathrm{RFP}^{+}\right)$per cell in each condition were quantified. (E) Effects of HDE on apoptosis at $24 \mathrm{~h}$. $(\mathrm{F}, \mathrm{G})$ HUVECs were incubated with $50 \mu \mathrm{g} / \mathrm{ml}$ oxLDL or Dil-labeled oxLDL in the absence or presence of $30 \mu \mathrm{g} / \mathrm{ml}$ HDE. After $48 \mathrm{~h}$, impaired autophagy was rescued by HDE and the amount of oxLDL contents was quantified by Dil intensity (scale bar: $20 \mu \mathrm{m}$ ). Results are presented as mean \pm SEM values. Statistical significance was assessed using Student's t-tests. $* * * P<0.001 ; * * P<0.01$. 
with yellow $\left(\mathrm{GFP}^{+} \mathrm{RFP}^{+}\right)$and their maturation into autolysosomes was expressed by red $\left(\mathrm{GFP}^{-} \mathrm{RFP}^{+}\right)$. The autophagy inducer, rapamycin, significantly increased the numbers of both yellow and red puncta. Bafilomycin A1 (BafA1) inhibits fusion of lysosomes and autophagosomes. BafA1 application resulted in accumulation of yellow puncta. Meanwhile, HDE increased red signals, suggesting that HDE treatment increased the autophagy flux (Fig. 2D). Additionally, the effect of HDE on apoptosis pathway was investigated by analyzing of several apoptosis markers including cleaved-PARP, cleaved-Caspase9 and cleaved-Caspase3 (Fig. 2E). HDE did not affect apoptotic signals at a concentration of up to $30 \mu \mathrm{g} / \mathrm{ml}$ at which autophagy was induced.

oxLDL induces ECs dysfunction and promotes endothelial lipid accumulation as well as leads to a deficiency of autophagy and accelerates atherosclerosis (20). The impaired autophagy in oxLDL-treated HUVECs was alleviated by HDE treatment (Fig. 2F). Under the same conditions, HDE reduced the fluorescence intensity of Dil-oxLDL by almost $40 \%$ (Fig. 2G), demonstrating that a lipid degradation was occurred during autophagy induction.

\section{HDE inhibited foam cell formation in oxLDL-induced} RAW264.7 macrophages via induction of autophagic activity When macrophages absorb oxLDL, they form foam cells and promote atherosclerosis. Therefore, it is essential to inhibit formation of macrophage foam cells to effectively treat atherosclerosis. To investigate the effects of $\mathrm{HDE}$ on oxLDL-induced foam cell formation, macrophages were stimulated with oxLDL and stained lipid droplets using Oil Red O. HDE significantly sup-

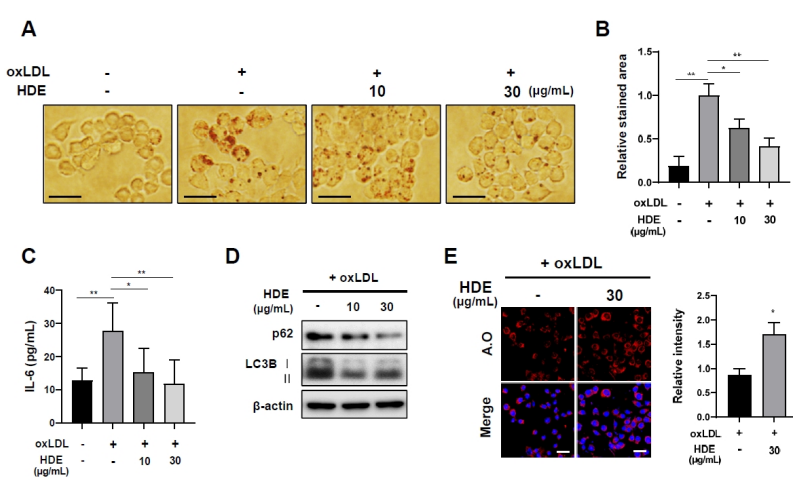

Fig. 3. HDE reduced formation of foam cells by regulating autophagy flux. (A) Representative images of Oil Red O staining of RAW264.7 cells treated with HDE along with oxLDL $(50 \mu \mathrm{g} / \mathrm{ml})$ for $48 \mathrm{~h}$ (scale bar: $20 \mu \mathrm{m})$. (B) The area of stained lipid droplets in each group were quantified using Image J. (C) HDE inhibited IL-6 production in oxLDLinduced RAW 264.7 in a concentration-dependent manner. Under the same conditions, (D) autophagy markers were monitored on western blots. (E) Representative images of acridine orange staining results. RAW264.7 macrophages were co-treated with oxLDL $(50 \mu \mathrm{g} / \mathrm{ml})$ and HDE $(30 \mu \mathrm{g} / \mathrm{ml})$ for $48 \mathrm{~h}$. Acidic vesicular organelles were detected (scale bar: $20 \mu \mathrm{m}$ ). Results are presented as mean \pm SEM values. Statistical significance was assessed using Student's $t$-tests. $* * * P<$ $0.001 ; * * P<0.01 ; * P<0.05$. pressed oxLDL-induced foam cell formation at $30 \mu \mathrm{g} / \mathrm{ml}$ concentration (Fig. 3A, B). Inflammatory cytokine production by macrophages also plays a critical role in the formation of foam cells. HDE inhibited IL-6 secretion of oxLDL-induced marcrophage measured with an ELISA kit (Fig. 3C). Previous study has shown that inhibition of autophagy leads to lipid accumulation in oxLDL-treated macrophage cells (21). Our results demonstrated that HDE upregulated the conversion of LC3, while p62 levels were decreased in oxLDL stimulated marcrophage (Fig. 3D).

Autophagy is highly associated with lysosome activity (22). Therefore, the effect of HDE on lysosome activity was examined using the lysosomotropic fluorescent dye, acridine orange, which stains acidic organelles in a pH-dependent manner. In the presence of oxLDL, HDE increased fluorescence intensity in RAW264.7 cells to about 1.5 times than that of the control. These results are consistent with the above results that HDE exhibits the autophagy induction activity (Fig. 3E).

\section{HDE attenuated formation of atherosclerotic plaque in ApoE $^{-1-}$ mice}

To examine the role of $\mathrm{HDE}$-induced autophagy on atherosclerotic plaque formation in vivo, $A p o E^{-1-}$ mice were fed a high cholesterol diet $(\mathrm{HCD})$. Mice were randomly divided into three groups and each was injected via the intraperitoneal route with vehicle or rapamycin $(10 \mathrm{mg} / \mathrm{kg})$ or HDE $(50 \mathrm{mg} / \mathrm{kg})$ every $2 \mathrm{~d}$ for 8 weeks. There were no differences in body weight among groups.
A

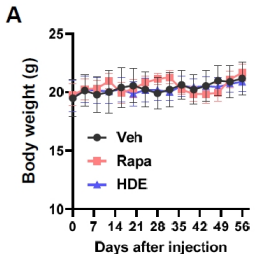

C

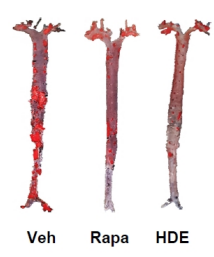

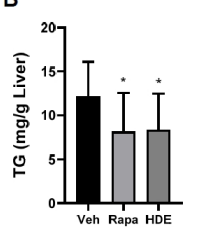

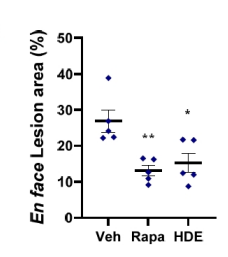

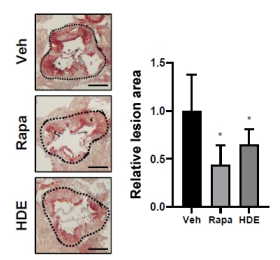

E

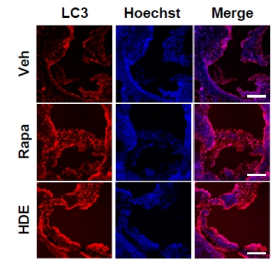

Fig. 4. $\mathrm{HDE}$ ameliorated atherosclerotic plaques in $A p o E^{-1-}$ mice model. (A) Schematic diagram of experimental design of $A p o E^{-1-}$ mice on a high cholesterol diet. Body weight of $\mathrm{ApoE}^{-/-}$mice was measured after injection $(n=5)$. There were no significant differences in average body weight after injection of vehicle, rapamycin $(10 \mathrm{mg} / \mathrm{kg})$, or HDE (50 mg/ $\mathrm{kg}$ ) during the 60-day period (29 treatments). (B) Accumulation of triglycerides in the liver tissue in each group was quantified. (C) Representative en face images of whole aortas from three treatment groups. Lesions in total aortas were investigated using Oil Red O staining. (D) Representative images of consecutive sections of an aortic sinus (stained with Oil Red O) (scale bar: $50 \mu \mathrm{m}$ ). (E) Representative images of consecutive aortic sinus sections immunostained with anti-LC3B antibody (scale bar : $100 \mu \mathrm{m}$ ). Results are presented as mean \pm SEM values. Statistical significance was assessed using Student's t-tests. ${ }^{*} P \mathrm{P}<0.01 ; * \mathrm{P}<0.05$. 
This result indicated the non-toxic effects of HDE on mouse viability (Fig. 4A).

Triglyceride (TG)-rich lipoproteins trigger atherosclerotic cardiovascular disease. Notably, HDE-treated mice ( $8.4 \mathrm{mg} / \mathrm{g}$ Liver) showed about 1.45 times less concentration of liver TG than that of the control (12.2 mg/g Liver) (Fig. 4B).

To investigate the anti-atherogenic effects of HDE in the aorta, we isolated whole aortas of the $A p o E^{-I-}$ mice and detected aortic atherosclerotic lesions stained with Oil Red O. Supplementation with $\mathrm{HDE}(15.3 \%)$ reduced atherosclerotic plaque burden by about $10 \%$ in the total aortas compared with HCD-fed control group (26.9\%) (Fig. 4C). The relative lesions of the aortic sinus were reduced by approximately 1.5 -fold in HDE-injected mice compared to vehicle (Fig. 4D).

We also stained aortic sinus sections with LC3 to examine whether HDE induced autophagy in atherosclerotic lesions. The LC3 staining results indicated that autophagy levels were increased in the HDE group, compared with the vehicle group (Fig. 4E). These results indicated that HDE had inhibitory effects on atherosclerotic plaque formation through increased autophagy activity in $A p o E^{-/-}$mice.

\section{DISCUSSION}

This study found that HHP under conditions of 550 MPa for 5 minutes efficiently killed microorganisms and increased extraction efficiency of raw danshen (Fig. 1). Typically, prokaryotes are more resistant to HHP than eukaryotes (23), and our results are consistent with this finding. HHP technology can be used to safely sterilize food, efficiently extract active ingredients, and be widely applied in medicinal plant industry.

We also investigated the effects of HDE on autophagy induction activity to relieve atherosclerosis. When ECs function normally, autophagy in this cell type is involved in vascular lipid homeostasis (24) that can protect blood vessels against atherosclerotic lesion formation. However, endothelial dysfunction contributes to atherosclerosis (25) and mouse models of atherosclerosis reveal that deficiencies in endothelial autophagy stimulate plaque formation (26). Thus, autophagy induction in ECs is a critical point in vascular-related disease. We found that HDE treatment $(30 \mu \mathrm{g} / \mathrm{ml})$ inhibited cell proliferation and induced autophagy in HUVECs, without toxic effects (Fig 2A-E). However, because danshen is known to induce apoptosis in cancer cells (12), HDE may trigger apoptosis at high concentrations. In addition, HDE reduced Dil-oxLDL contents, suggesting that HDE exhibited lipid degradation via autophagy induction (Fig. 2F, G). Cryptotashinone (MW 296.4), one of the active principles of danshen, has autophagic and anti-atherosclerotic activities $(27,28)$. Therefore, we assumed that cryptotashinone could be responsible for biological activities of HDE.

This study revealed that HDE prevented foam cell formation by inhibiting macrophage oxLDL absorption and simultaneously increasing lysosomal activity (Fig. 3). Additionally, we found that administration of HDE reduced atherosclerotic plaque for- mation in the $A p o E^{-/-}$mouse model (Fig. 4). This result indicated, for the first time, that danshen suppressed atherosclerosis via an autophagy-inducing mechanism.

In summary, this study demonstrated that HHP was an effective method for raw danshen processing. The HHP treated raw danshen extract, $\mathrm{HDE}$, suppressed foam cell formation via enhancement of autophagy activity. We also found anti-atherosclerotic activity of HDE at the in vivo level. Taken together, these results suggested that HDE could be developed as a potential naturally-derived alternative medicinal substance to ameliorate atherosclerosis.

\section{MATERIALS AND METHODS}

\section{Preparation of danshen using HHP treatment}

Raw danshen was obtained from a local agricultural products market (Incheon, Korea). Raw danshen (50 g) was packaged in a pouch and heat-sealed. Packaged danshen was subjected to $\mathrm{HHP}$ at $550 \mathrm{MPa}$ for $5 \mathrm{~min}$ at $25^{\circ} \mathrm{C}$ using a pilot-scale HHP unit (5.0-L capacity, HHP-600, Baotou Kefa Co., Ltd., Inner Mongolia, China).

\section{Microbiological analysis}

Total aerobic counts and yeasts and molds of raw danshen and HHP-treated danshen were investigated. Each sample (20 g) was placed in a sterile filter bag with $60 \mathrm{ml} 0.1 \%$ buffered peptone water and stomached for 2 min using a stomacher (MIX 2, AES Laboratories, Combourg, France). Samples were serially diluted using saline solution; 1-ml samples of the diluted solution were then plated onto triplicate plates of nutrient agar for total aerobic counts and onto potato dextrose agar (acidified to $\mathrm{pH} 3.5$ with $10 \%$ tartaric acid) for yeasts and molds. Total aerobic counts of colonies were counted after incubation at $37^{\circ} \mathrm{C}$ for $24 \mathrm{~h}$. Colonies of yeast and molds were counted after incubation at $25^{\circ} \mathrm{C}$ for $3 \mathrm{~d}$.

\section{Extraction}

Samples (50 g) of raw danshen and HHP-treated danshen were extracted for $4 \mathrm{~h}$ with $500 \mathrm{ml}$ boiling water. The extract was filtered two times and then fractionated with ethyl acetate. Collected fractions were vacuum-dried. To compare extraction yield (\%) according to HHP treatment, each extraction yield was calculated based on weight [(weight of extract obtained after vacuum drying $(\mathrm{g})) /($ weight of raw material $(50 \mathrm{~g})) \times 100]$. For in vitro experiments, vacuum-dried samples were used by DMSO ( $\mu \mathrm{g} / \mathrm{ml})$.

\section{Cell culture}

HUVECs were cultured in EBM-2 (Lonza) containing supplements. RAW264.7 cells were maintained in DMEM (Gibco) with 10\% FBS (Gibco). All cells were incubated at $37^{\circ} \mathrm{C}$ in a humidified $5 \% \mathrm{CO}_{2}$ atmosphere at $\mathrm{pH} 7.4$. 


\begin{abstract}
MTT assay
HUVECs were seeded into 96-well plates $\left(3 \times 10^{3}\right.$ cells/well) and maintained overnight. The cells were then treated with various doses of HDE. Cell proliferation was measured in triplicate using 3-(4,5-dimethylthiazol-2-yl)-2,5-diphenyltetrazolium bromide (MTT; Sigma-Aldrich) at $0.4 \mathrm{mg} / \mathrm{ml}$ (final concentration). The MTT formazan product in each well was dissolved in DMSO and absorbance was measured at a 595-nm wavelength with a microplate reader.
\end{abstract}

\section{mRFP-GFP-LC3B plasmid transfection}

HUVECs were transfected with mRFP-GFP-LC3B plasmid using lipofectamine RNA iMAX transfection reagent (Invitrogen) for $24 \mathrm{~h}$. The cells were then treated with DMSO, rapamycin, Baf A1, or HDE. Nuclei were stained with Hoechst 33432 and cells were fixed with $4 \%$ formaldehyde. Images were obtained using an LSM880 confocal microscope at $400 \times$ magnification. Red and green puncta were counted.

\section{Western blot}

Cells were seeded onto 12-well plates. Soluble proteins were harvested using $2 \times$ SDS lysis buffer $(0.12 \mathrm{M}$ Tris-Cl, $\mathrm{pH}$ 6.8, $3.3 \%$ SDS, $10 \%$ glycerol, $3.1 \%$ DTT). The lysates were separated using sodium dodecyl sulfate polyacrylamide gel electrophoresis (SDS-PAGE; resolving buffer 1.5 M Tris-Cl, $\mathrm{pH} 8.8$, stacking buffer $0.5 \mathrm{M}$ Tris-Cl, pH 6.8). The gels were transferred to polyvinylidene difluoride membranes (BioRad). Incubate membrane with diluted antibody (anti-p62 (610833, BD Biosciences), antiSQSTM1/p62 (5114s, CST), anti-LC3B (2775s, CST), anti-PARP (9542s, CST), anti-Caspase3 (9662s, CST), anti-Caspase9 (9502s, CST), anti-actin (ab6276, Abcam)) in $3 \%(\mathrm{w} / \mathrm{v})$ skim milk or $5 \%(\mathrm{w} / \mathrm{v})$ BSA overnight at $4^{\circ} \mathrm{C}$. Rabbit and mouse secondary antibodies (1:3000 v/v, GE Healthcare) were treated in $3 \%$ $(\mathrm{w} / \mathrm{v})$ skim milk for $1 \mathrm{~h}$ at $25^{\circ} \mathrm{C}$. Immunolabeling was detected using an ECL substrate (BioRad) according to the manufacturer's instructions and a ChemiDoc XRS + imaging system (BioRad).

\section{Foam cell formation}

RAW264.7 macrophage cells were seeded in 6-well plates and grown in medium containing $50 \mu \mathrm{g} / \mathrm{ml}$ oxLDL (Invitrogen). After a 48-h incubation, the cells were fixed and stained with Oil Red O to confirm that the normal RAW264.7 changed into foam cell morphology. Cells were examined under a microscope, and images were obtained.

\section{ELISA}

RAW264.7 cells were seeded in 24-well plates $\left(4 \times 10^{5}\right.$ cells/ well) and cultured overnight. The cells were pre-treated with HDE for $1 \mathrm{~h}$ and then treated with $50 \mu \mathrm{g} / \mathrm{ml}$ oxLDL for $24 \mathrm{~h}$. The culture supernatants were collected for the measurement of IL-6 levels using the ELISA kit (88-7064, Invitrogen) according to the manufacturer's instructions.

\section{Acridine orange staining}

RAW264.7 macrophage cells were seeded in 6-well plates and grown in medium containing $50 \mu \mathrm{g} / \mathrm{ml}$ oxLDL (Invitrogen) with or without HDE. After a 48-h incubation, the cells were stained with acridine orange $(2.5 \mu \mathrm{g} / \mathrm{ml})$ and Hoecsht 33432 solution at room temperature for $20 \mathrm{~min}$. Cells were fixed with $4 \%$ PFA and mounted on glass slides. The images were captured under a Zeiss LSM 880 confocal microscope.

\section{Animal experiments}

Animal studies were approved by the Institutional Animal Care and Use Committee of Yonsei University (IACUC-A-201904883-01). ApoE ${ }^{-/-}$mice were provided by Dr. Goo Taeg Oh, Ewha Womans University. They were housed under a 12-h daynight cycle with free access to water and food in a specificpathogen-free system. The mice were fed a high cholesterol diet from 6 weeks of age to the end of the experiment.

\section{Triglyceride assay}

Liver tissues were homogenized using 5\% NP-40 and supernatants were separated. Triglyceride content was measured using Triglyceride Quantification Kit (ab65336, Abcam) following the manufacturer's instructions.

\section{Atherosclerosis analysis}

Mice were euthanized with $\mathrm{CO}_{2}$ gas, perfused with PBS through the left ventricle, and hearts and aortas were isolated. Aortas were cut from the proximal ascending region to the bifurcation of the iliac artery. Adventitial fat was removed, and each aorta was dissected longitudinally and pinned onto black silicone plates. After fixation with $10 \%$ neutral buffered formalin, the tissue samples were stained with Oil Red O overnight and washed with PBS. The base of the heart containing the aortic root was embedded in OCT medium, frozen at $-80^{\circ} \mathrm{C}$, and serial sections were collected. Atherosclerotic lesions were stained using Oil Red $\mathrm{O}$ stain and quantified using Image J software. For fluorescence immunohistochemistry, the sections were blocked with $10 \%$ BSA for 30 min, then stained with primary anti-LC3 antibody (ab48394) overnight at $4^{\circ} \mathrm{C}$. After washing three times with PBS, the sections were stained with Alexa Fluor 594-conjugated goat anti-rabbit IgG for $1 \mathrm{~h}$. The sections were then stained with Hoechst 33432 for 5 min and washed with PBS. The coverslips were mounted onto glass slides with fluorescent mounting medium and the results were analyzed.

\section{Statistical analysis}

All results were presented as mean \pm standard error of the mean (SEM) values using Prism 5.0 (GraphPad Software). Statistical significance was determined using Student's t-tests.

\section{ACKNOWLEDGEMENTS}

This work was supported by grants from the National Research Foundation of Korea, funded by the Korean government (MSIP; 
2015K1A1A2028365, 2015M3A9B6027818, 2016K2A9A1A03 904900, 2018M3A9C4076477), the Brain Korea 21Plus Project in the Republic of Korea, and ICONS (Institute of Convergence Science), Yonsei University.

\section{CONFLICTS OF INTEREST}

The authors have no conflicting interests.

\section{REFERENCES}

1. Benjamin EJ, Muntner P, Alonso A et al (2019) Heart disease and stroke Statistics-2019 update a report from the American Heart Association. Circulation 139, e56-e528

2. Glass CK and Witztum JL (2001) Atherosclerosis: the road ahead. Cell 104, 503-516

3. Moore KJ, Sheedy FJ and Fisher EA (2013) Macrophages in atherosclerosis: a dynamic balance. Nat Rev Immunol 13, 709-721

4. Luo H, Wang J, Qiao C, Ma N, Liu D and Zhang W (2015) Pycnogenol attenuates atherosclerosis by regulating lipid metabolism through the TLR4-NF-кB pathway. Exp Mol Med 47, e191-e191

5. San Martin M, Barbosa-Cánovas G and Swanson B (2002) Food processing by high hydrostatic pressure. Crit Rev Food Sci Nutr 42, 627-645

6. Elamin WM, Endan JB, Yosuf YA, Shamsudin $R$ and Ahmedov A (2015) High pressure processing technology and equipment evolution: a review. J Eng Sci Technol Rev 8, 75-83

7. Rendueles E, Omer M, Alvseike O, Alonso-Calleja C, Capita R and Prieto M (2011) Microbiological food safety assessment of high hydrostatic pressure processing: A review. LWT - Food Science and Technology 44, 1251-1260

8. Serment-Moreno V, Barbosa-Canovas G, Torres JA and Welti-Chanes J (2014) High-pressure processing: kinetic models for microbial and enzyme inactivation. Food Eng Rev 6, 56-88

9. Garcia AF, Butz P and Tauscher B (2001) Effects of highpressure processing on carotenoid extractability, antioxidant activity, glucose diffusion, and water binding of tomato puree (Lycopersicon esculentum Mill.). J Food Sci 66, 10331038

10. Khan SA, Aslam R and Makroo HA (2019) High pressure extraction and its application in the extraction of bio-active compounds: A review. J Food Proc Eng 42, e12896

11. Cheng TO (2007) Cardiovascular effects of Danshen. Int J Cardiol 121, 9-22

12. Chen X, Guo J, Bao J, Lu J and Wang Y (2014) The anticancer properties of Salvia miltiorrhiza Bunge (Danshen): a systematic review. Med $\operatorname{Res} \operatorname{Rev} 34,768-794$

13. Chun-Yan S, Qian-Liang M, Rahman K, Ting H and Lu-Ping
Q (2015) Salvia miltiorrhiza: traditional medicinal uses, chemistry, and pharmacology. Chin J Nat Med 13, 163182

14. Kundu M and Thompson CB (2008) Autophagy: basic principles and relevance to disease. Annu Rev Pathol 3, 427-455

15. Martinet W, Agostinis $P$, Vanhoecke B, Dewaele M and De Meyer GR (2009) Autophagy in disease: a double-edged sword with therapeutic potential. Clin Sci 116, 697-712

16. Liao X, Sluimer JC, Wang $Y$ et al (2012) Macrophage autophagy plays a protective role in advanced atherosclerosis. Cell Metab 15, 545-553

17. Shao B, Han B, Zeng Y, Su D and Liu C (2016) The roles of macrophage autophagy in atherosclerosis. Acta Pharmacol Sin 37, 150-156

18. Prasad KN, Yang E, Yi C, Zhao M and Jiang Y (2009) Effects of high pressure extraction on the extraction yield, total phenolic content and antioxidant activity of longan fruit pericarp. Innov Food Sci Emerg Technol 10, 155-159

19. Yoshii SR and Mizushima N (2017) Monitoring and measuring autophagy. Int J Mol Sci 18, 1865

20. Zhang CP, Ding XX, Tian T et al (2020) Impaired lipophagy in endothelial cells with prolonged exposure to oxidized low-density lipoprotein. Mol Med Rep 22, 2665-2672

21. Gu HF, Li HZ, Tang YL, Tang XQ, Zheng XL and Liao DF (2016) Nicotinate-curcumin impedes foam cell formation from THP-1 cells through restoring autophagy flux. PLoS One 11, e0154820

22. Yim WWY and Mizushima N (2020) Lysosome biology in autophagy. Cell Discov 6, 1-12

23. Zhang $\mathrm{ZH}$, Wang $\mathrm{LH}$, Zeng $X \mathrm{~A}$, Han $\mathrm{Z}$ and Brennan CS (2019) Non-thermal technologies and its current and future application in the food industry: a review. Int J Food Sci Technol 54, 1-13

24. Torisu K, Singh KK, Torisu T et al (2016) Intact endothelial autophagy is required to maintain vascular lipid homeostasis. Aging Cell 15, 187-191

25. Kim SM, Huh JW, Kim EY et al (2019) Endothelial dysfunction induces atherosclerosis: increased aggrecan expression promotes apoptosis in vascular smooth muscle cells. BMB Rep 52, 145-150

26. Kheloufi M, Vion AC, Hammoutene A et al (2018) Endothelial autophagic flux hampers atherosclerotic lesion development. Autophagy 14, 173-175

27. Xu Z, Jiang H, Zhu Y et al (2017) Cryptotanshinone induces ROS-dependent autophagy in multidrug-resistant colon cancer cells. Chem Biol Interact 273, 48-55

28. Liu Z, Xu S, Huang X et al (2015) Cryptotanshinone, an orally bioactive herbal compound from Danshen, attenuates atherosclerosis in apolipoprotein E-deficient mice: role of lectin-like oxidized LDL receptor-1 (LOX-1). Br J Pharmacol $172,5661-5675$ 